1 Submitted to Powder Technology, Sep. 6th, 2020

\title{
2 A Novel CFD-DEM Coarse-Graining Method Based on the 3 Voronoi Tessellation
}

4 Hanqiao Che ${ }^{1}$, Catherine O'Sullivan ${ }^{1}$, Adnan $\mathrm{Sufian}^{2}$, Edward Smith ${ }^{3}$

5 1. Department of Civil and Environmental Engineering, Imperial College London, South Kensington

6 Campus, SW7 2AZ, UK

7 2. School of Civil Engineering, The University of Queensland, St Lucia, 4072, Australia

8 3. Department of Mechanical and Aerospace Engineering, Brunel University London, Uxbridge,

$9 \quad$ Middlesex UB8 3PH, UK

10 E-mail address: h.che@imperial.ac.uk (Hanqiao Che)

\begin{abstract}
11 Abstract
12 In unresolved flow CFD-DEM simulations, the porosity values for each CFD cell are determined using

13 a coarse-graining algorithm. While this approach enables coupled simulations of representative numbers

14 of particles, the influence of the porosity local to the particles on the fluid-particle interaction force is

15 not captured. This contribution considers a two-grid coarse-graining method that determines a local
\end{abstract}

16 porosity for each particle using a radical Voronoi tessellation of the system. A relatively fine, regular

17 point cloud is used to map these local porosity data to the CFD cells. The method is evaluated using two

18 different cases considering both disperse and tightly packed particles. The data show that the method

19 conserves porosity data, is reasonably grid-independent and can generate a relatively smooth porosity

Formatted: Font colour: Text 1

20 field. The new method can more accurately predict the fluid-particle interactive force for polydisperse 
1 particle system than alternative methods that have been implemented in unresolved CFD-DEM codes.

2

3 Keywords: Radical Voronoi tessellation; CFD-DEM; Coarse-Graining; Fluid-particle interactive force. 


\section{1. Introduction}

2 Coupled computational fluid dynamics - discrete element method (CFD-DEM) simulations can provide

3 valuable insight across a range of applications including the granulation of pharmaceutical products [1]

4 mining / mineral extraction [2] debris flows and internal erosion of dams and flood embankments [3].

5 A common approach to CFD-DEM is based on an Eulerian-Lagrangian framework, where the fluid

6 phase is simulated using a fixed Eulerian grid with grid spacing that is larger than the particles

7 (unresolved approach) or measurably smaller than the particles (resolved approach) [4]. One resolved

8 CFD-DEM approach is the immersed boundary method (IBM) [5] in which the points on the surfaces

9 of particles are inserted in the fluid region as additional no-slip boundary conditions, and a source term

10 representing the fluid-particle interaction is added into the Navier-Stokes equations to describe the fluid

11 flow that is solved on the fixed Eulerian grid. In unresolved CFD-DEM, a porous version of the Navier-

12 Stokes equations is solved [6]: the presence of particles in the fluid is accounted for by including the

13 porosity value. The particle-fluid interaction forces are estimated by empirical drag correlations[7-10]

14 and included in the CFD equations as a source term. On the DEM side the particle-fluid interaction force

15 is considered alongside the inter-particle contact forces and any gravitational forces [4, 11] when

16 determining the resultant forces acting on the particles. CFD-DEM is a coupled simulation process and

17 the DEM and CFD solvers can be executed either concurrently or sequentially. After a specified number

18 of time-steps, relevant variables such as porosity, drag force and velocity of each phase are exchanged

19 between both solvers.
Formatted: Font colour: Text 1

Formatted: Font colour: Text 1

Formatted: Font colour: Text 1

Formatted: Font colour: Text 1

Formatted: Font colour: Text 1

Formatted: Font colour: Text 1

Formatted: Font colour: Text 1

Formatted: Font colour: Text 1

Formatted: Font colour: Text 1

Formatted: Font colour: Text 1

Formatted: Font colour: Text 1

Formatted: Font colour: Text 1

Formatted: Font colour: Text 1

Formatted: Font colour: Text 1

Formatted: Font colour: Text 1

Formatted: Font colour: Text 1

Formatted: Font colour: Text 1 
1 In an unresolved CFD-DEM simulation, the coarse-graining stage is very important. In this stage, the

2 relevant coupling data, including the porosity, solid velocity and the momentum source terms for each

3 CFD cell, are determined. The coarse-graining approach used must satisfy several requirements $[12,13]$

4 most significantly it must (1) conserve relevant physical quantities such as the total particle volume; (2)

5 be able to achieve relatively grid-independent results, and (3) be able to produce smooth coarse-grained

6 fields without large jumps existing between the neighbouring CFD cells.

8 The simplest coarse-graining method adopted in unresolved CFD-DEM is the particle centroid method

9 (PCM) [14]. PCM estimates the volume of the particles within a CFD cell by summing up the volume

10 of the particles whose centres are located in that cell. This method is fast but can be only applied in

11 cases where the cell-to-particle size ratio is large. Large jumps in the porosity values may occur and

12 lead to big oscillations and high-frequency peaks in the simulation results [15].

13

14 In the divided method (DM) $[11,16]$ the proportion of each particle is assigned to the CFD cell is based

15 on the actual overlap volume between the particle and the cell boundary; this is more accurate than the

16 PCM approach. There are many strategies to estimate the overlap volume. For example, the particle can

17 be first resolved by a series of distributed marker points to evenly apportion the particle's volume to all

18 cells that are (partly) covered, and then the relevant particle sub-region is assigned to the CFD cell using

19 PCM $[11,17]$. Where the particle size is approximately equal to or even larger than the grid size, the

20 large particles can be represented by a number of small particles [18]. DM can generally eliminate the

21 jumps in the porosity field between the neighbouring CFD cells and provides smoother porosity field
Formatted: Font colour: Text 1

Formatted: Font colour: Text 1

Formatted: Font colour: Text 1

Formatted: Font colour: Text 1

Formatted: Font colour: Text 1

Formatted: Font colour: Text 1

Formatted: Font colour: Text 1

Formatted: Font colour: Text 1

Formatted: Font colour: Text 1

Formatted: Font colour: Text 1

Formatted: Font colour: Text 1

Formatted: Font colour: Text 1

Formatted: Font colour: Text 1 
1 than PCM.

3 The porous sphere [19] or porous cube [20] methods represent the particles as porous elements and then

4 distribute the volume of the elements to the CFD cells within a region that is larger than the particle size

5 to avoid extreme porosity values in the CFD cells. To further improve the smoothness of the porosity

6 field, the statistical kernel method [21] or the diffusion-based coarse-graining method [12] can be used.

7 These methods apply a statistical kernel or a diffusion source to the centre of each particle, and the volume of the particle is distributed to the calculation domain. In these approaches, a portion of the

11 than the particle diameter [12]

12

13 The general approach adopted in the above-mentioned methods is to firstly bin or assign a proportion

14 of each particle's volume to the predefined fluid cells, and then calculate the porosity of each fluid cell.

15 Accordingly, the porosity data are "grid-dependent". The CFD grid employed should be chosen to maintain the smoothness of the porosity field and the resolution of fine-scale fluid features is limited

17 [22]. A two-grid method has been proposed [22, 23] in which the porosity is calculated based on a

18 coarse grid (grid size larger than particles) and mapped to a finer grid that is used by the CFD solver.

19 However, a key issue with this type of two-grid method is how to select an appropriate grid size. The

20 shape and size of the coarse grid selected seems arbitrary, for example, a cube with three times the

21 particle diameter as the length was used by Deb \& Tafti [22] and no data to support decisions around the
Formatted: Font colour: Text 1

Formatted: Font colour: Text 1

Formatted: Font colour: Text 1

Formatted: Font colour: Text 1

Formatted: Font colour: Text 1

Formatted: Font colour: Text 1

Formatted: Font colour: Text 1

Formatted: Font colour: Text 1

Formatted: Font colour: Text 1

Formatted: Font colour: Text 1

Formatted: Font colour: Text 1

Formatted: Font colour: Text 1

Formatted: Font colour: Text 1

Formatted: Font colour: Text 1

Formatted: Font colour: Text 1

Formatted: Font colour: Text 1

Formatted: Font colour: Text 1 
1 choice of grid spacing is available.

\section{$<$ Fig. 1 Schematic of the conventional and radical Voronoi tessellation $>$}

3 A Voronoi tessellation can be used to identify a local porosity for each particle in the system. A Voronoi

4 tessellation divides the particle domain into a unique space filling set of polyhedral cells (Fig 1(a)). In

5 case of monodisperse particle systems, only the coordinates of the particle centre are used as the input;

the planes of the polyhedral Voronoi cells are located at the midpoints of the lines (Delaunay tetrahedra

7 edges) connecting adjacent particles. For polydisperse particle systems, if this basic partitioning

8 approach is adopted the polyhedral planes defining the Voronoi cells can intercept the particles. The

9 radical Voronoi tessellation was developed to solve this problem [24]. In a radical Voronoi tessellation,

10 the space between particles is partitioned based on radical planes, i.e., the planes formed between pairs

11 of particles for which the tangent lines from a point in the plane to both particles are of equal length.

12 Voronoi tessellations can be implemented in DEM codes using existing libraries [25]. This approach

13 has been used in previous DEM simulations to quantify the void space [26]. By comparing with IBM

14 simulations, Knight et al. [27] showed that, in principle, this local porosity could be used to calculate

15 the drag force on individual particles in polydisperse systems. However, there are no published studies

16 documenting a successful implementation of this approach in an unresolved CFD-DEM simulation

17 framework.

18

19 The objective of this paper is to introduce a new CFD-DEM coarse-graining method and evaluate its

20 performance. Following an overview of unresolved CFD-DEM, Section 3 describes how the local

21 porosity of each particle is calculated based on the particle volume and the volume of the surrounding
Formatted: Font colour: Text 1

Formatted: Font colour: Text 1

Formatted: Font colour: Text 1

Formatted: Font colour: Text 1

Formatted: Font colour: Text 1

Formatted: Font colour: Text 1

Formatted: Font colour: Text 1

Formatted: Font colour: Text 1

Formatted: Font colour: Text 1

Formatted: Font colour: Text 1 
1 Voronoi cells, and then mapped to the CFD grid using a point cloud method. In Section 4 two validation

2 cases which represent typical applications of CFD-DEM are considered, and the simulation data

3 generated are compared to previously generated IBM data from [27] as well as to appropriate

4 experimental data.

\section{2. Governing equations in CFD-DEM}

6 CFD-DEM typically involves coupling an established DEM code with an established CFD solver [28-

7 30]. In the current study, the open-source code CPL-Library [31] was used to couple the open-source

8 codes LAMMPS and OpenFOAM. All coupling in CPL-Library is handled through shared libraries

9 which use the message passing interface (MPI) to facilitate information exchange between software.

10 Fig. 2 shows the flowchart of the CFD-DEM coupling in CPL-Library; the Navier-Stokes equations are

11 solved in the CFD solver, while the DEM solver simulates the motion of and interaction between the

12 particles.

13

14 In unresolved CFD-DEM simulations, the porosity $\left(\varepsilon_{f}\right)$ is included in the system of equations considered

15 in the CFD solver. For incompressible flow, the volume-averaged continuity and momentum equations

16 are written as

$$
\frac{\partial \varepsilon_{f}}{\partial t}+\nabla \cdot\left(\varepsilon_{f} \mathbf{u}_{f}\right)=0
$$

$18 \quad \frac{\partial}{\partial t}\left(\varepsilon_{f} \rho_{f} \mathbf{u}_{f}\right)+\nabla \cdot\left(\varepsilon_{f} \rho_{f} \mathbf{u}_{f} \otimes \mathbf{u}_{f}\right)=-\varepsilon_{f} \nabla p+\varepsilon_{f} \nabla \cdot \boldsymbol{\tau}-\mathbf{S}_{p f}+\varepsilon_{f} \rho_{f} \mathbf{g}$,

19 where $\rho_{f}$ and $\mathbf{u}_{f}$ are the fluid density and velocity, $\boldsymbol{\tau}$ is the viscous stress tensor, $\mathbf{g}$ is gravity, $\mathbf{S}_{p f}$ is the
Formatted: Font colour: Text 1

Formatted: Font colour: Text 1

Formatted: Font colour: Text 1

Formatted: Font colour: Text 1

Formatted: Font colour: Text 1

Formatted: Font colour: Text 1

Formatted: Font colour: Text 1

Formatted: Font colour: Text 1

Formatted: Font colour: Text 1

Formatted: Font colour: Text 1

Formatted: Font colour: Text 1

Formatted: Font colour: Text 1 
2 split-up into an implicit and an explicit part $[11,28]$ and is written as

3

$$
\mathbf{S}_{p f}=K_{p f} \mathbf{u}_{f}-K_{p f}\left\langle\mathbf{u}_{p}\right\rangle
$$

where $<\mathbf{u}_{\mathrm{p}}>$ is the cell-based ensemble-averaged particle velocity, $\mathbf{u}_{f}$ is discretised implicitly in the CFD

solver; the (scalar) coefficient $K_{p f}$ is a function of the drag force on particles, which is estimated by

$$
K_{p f}=\frac{\left|\sum_{i} \mathbf{F}_{d, i}\right|}{V_{\text {cell }}\left|\mathbf{u}_{f}-\left\langle\mathbf{u}_{p}\right\rangle\right|},
$$

$7 \quad$ where $\mathbf{F}_{d, i}$ is the drag force acting on a single particle $i$. There are many empirical drag correlations

In the DEM solver the motion of particle $i$ is calculated by the total force and torque acting on it:

$$
m_{i} \frac{\mathrm{d} \mathbf{u}_{i}}{\mathrm{~d} t}=m_{i} \mathbf{g}+\sum_{j=0}^{k}\left(F_{n, i j} \mathbf{n}+F_{t, i j} \mathbf{t}\right)+\mathbf{F}_{d, i}+\mathbf{F}_{p, i}+\mathbf{F}_{\tau, i}
$$

$$
\mathbf{I}_{i} \frac{d \omega_{i}}{d t}=\sum_{j=0}^{k}\left[\mathbf{r} \times\left(F_{t, i j} \mathbf{t}\right)\right]
$$

where $F_{n, i j}$ and $F_{t, i j}$ are the normal and tangential contact forces associated with contact between particles $i$ and $j, k$ is the total number of contacts involving particle $i, \mathbf{F}_{p, i}$ and $\mathbf{F}_{\tau, i}$ represent the pressure gradient force and the viscous force respectively, which are exerted by the surrounding fluid; $\mathbf{I}_{i}$ is the moment of inertia of the particle. The magnitudes of the contact force are calculated based on a spring-dashpot soft sphere contact model with a "history" effect [34]:
Formatted: Font colour: Text 1

Formatted: Font colour: Text 1

Formatted: Font colour: Text 1

Formatted: Font colour: Text 1

Formatted: Font colour: Text 1

Formatted: Font colour: Text 1

Formatted: Font colour: Text 1

Formatted: Font colour: Text 1

Formatted: Font colour: Text 1

Formatted: Font colour: Text 1

Formatted: Font colour: Text 1

Formatted: Font colour: Text 1

Field Code Changed

Formatted: Font colour: Text 1

Formatted: Font colour: Text 1

Formatted: Font colour: Text 1

Formatted: Font colour: Text 1

Formatted: Font colour: Text 1

Formatted: Font colour: Text 1
Formatted: Font colour: Text 1

Formatted: Font colour: Text 1 


$$
F_{t}=\left\{\begin{array}{c}
k_{t} \int_{t 0}^{t} \Delta u_{t} \mathrm{~d} t+c_{t} \Delta u_{t},\left|F_{t}\right| \leq \mu F_{n} \\
\mu F_{n},\left|F_{t}\right| \geq \mu F_{n},
\end{array}\right.
$$

7 The coupling loop proposed here includes 4 main steps: Step 1: The DEM and CFD solver are executed and 4 are described in the following sections.

concurrently; Step 2: A radical Voronoi tessellation is carried out based on the current particle positions so that the local particle porosity can be evaluated; Step 3: The fluid fields are mapped to each particle and the drag force acting on each particle is calculated; Step 4: The particle porosity, velocity and the drag force are mapped to the CFD side; Step 5: Based on the updated fluid information for coupling,
Formatted: Font colour: Text 1

Formatted: Font colour: Text 1

Formatted: Font colour: Text 1

Formatted: Font colour: Text 1 3. Two-grid, Voronoi tessellation-based coarse-graining method

15 The two-grid coarse-graining method proposed here comprises two main steps: (1) Radical Voronoi

16 tessellation of the particle system to assign a local porosity to each particle; (2) Field mapping to the

17 CFD grid using a point cloud.

Formatted: Font colour: Text 1 


\section{$1 \quad 3.1$ Voronoi tessellation of the particle system}

$2<$ Fig. 3 Schematic of the Voronoi tessellation of the particle system (the Voronoi cells are built in 3D

3 space) $>$

4 Fig. 3 is a schematic of the Voronoi tessellation of the particle system. In the case of a uniformly

5 distributed particle system in a predefined domain, use of a Voronoi tessellation to obtain representative

6 local porosity values is straightforward (see Fig. 3a). However, in many applications, such as fluidised

7 beds, there are regions with dilute particle flow. In these regions the Voronoi cells become large and

8 would give unreasonable estimates of the local porosity. To resolve this problem, a bounding cuboid

9 which is larger than the particle is generated. If the boundaries of the particle's Voronoi cell stretches

10 beyond the initial bounding cuboid, they are replaced by the cuboid boundaries (see Fig. 3b). The size

11 of the bounding cuboid is controlled by a parameter $\left(\theta_{1}\right)$, which equals the cuboid-to-particle size ratio

$12\left(\theta_{1}=B / D\right)$. The cuboid bounding box gives an upper bound to the calculated porosity, for example, for

Formatted: Font colour: Text 1

$13 \theta_{1}=2,3$ and 4, the maximum local porosities for individual particles are $0.93,0.98$ and 0.99 , respectively.

14

15 The local porosity of an individual particle is simply the volume of the void space within the particle's

16 Voronoi cell divided by the total volume of the particle's Voronoi cell, i.e.,

$$
\varepsilon_{f, i}^{V}=\frac{V_{\text {voro }, i}-V_{p, i}}{V_{\text {voro }, i}}
$$

where $V_{p, i}$ and $V_{v o r o, i}$ are the volumes of particle $i$ and the surrounding Voronoi cell, the superscript $V$

19 represents the porosity is in a Voronoi cell.

20

21 Here the Voronoi tessellation of the particle system was carried out by adding voro++ library to 
1 LAMMPS as a user package $[25]$ and it can be compiled with the CPL-Library for the CFD-DEM

2 coupling.

\section{$3 \quad 3.2$ Field mapping using the point cloud}

4 As noted above, CFD-DEM requires a field mapping to exchange key information between the DEM

5 and CFD solvers. Due to the complex geometric structure of the Voronoi cells, the field mapping of the

6 particle data to the fluid grid is achieved by mapping data to a point cloud. This method uses a fine

7 uniform grid of discrete points (termed a point cloud) to identify (with some approximation /

8 discretization error) the region that is common to a particular Voronoi cell and the CFD cell that overlaps

9 it. As shown in Fig. 4, a regular grid of sampling points is generated that is evenly distributed across the

10 whole computational domain. The density of the point cloud is denoted by the parameter $\theta_{2}$, which

11 equals the ratio of the smallest particle diameter and the point cloud grid spacing $\left(\theta_{2}=D_{\min } / S_{p c}\right)$. A

12 systematic inspection of the sampling points in the cloud (with given position vectors) is carried out to

13 identify which particle Voronoi cell and which CFD cell contain the point. This exercise generates two

14 one-dimensional arrays (vectors); these store (1) the number of sampling points lying within Voronoi

15 cell $i\left(N_{i}^{\text {total }}\right)$ and (2) the number of sampling points in CFD cell $n\left(N_{n}^{\text {total }}\right)$. Then a 2D sparse array that

16 stores the number of sampling points shared by specific Voronoi cell $i$ and CFD cell $n\left(N_{i, n}^{\text {share }}\right)$ is populated.

17 The arrays $N_{i}^{\text {total }}, N_{n}^{\text {total }}$ and $N_{i, n}^{\text {share }}$ are updated whenever the Voronoi cells are rebuilt and are used in the

18 subsequent field mapping procedures.

$<$ Fig. 4 Schematic of the field mapping method using point cloud $>$

20 Fig. 4a shows the schematic of the forward mapping step, i.e., the mapping from a "source" Voronoi
Formatted: Font colour: Text 1

Formatted: Font colour: Text 1

Formatted: Font colour: Text 1

Formatted: Font colour: Text 1

Formatted: Font colour: Text 1

Formatted: Font colour: Text 1 
1 cell to the overlying "target" CFD cell. For a given porosity field $\varepsilon_{f i i}^{V}$ in the Voronoi polygon $i$, the

2 magnitude of the porosity mapped to target CFD cell $n$ is given by

3

$$
\varepsilon_{f, n}^{C}=\sum_{i=1}^{N_{p}}\left(\varepsilon_{f, i}^{V} \frac{N_{i, n}^{\text {share }}}{N_{n}^{\text {total }}}\right) \text {, }
$$

where $N_{p}$ equals the total number of particles in the simulation domain and the superscript $C$ indicates that the calculated porosity relates to the CFD grid. For the particle velocity, the mapping scheme is

6

$$
u_{s, n}^{C}=\frac{\sum_{i=1}^{N_{p}}\left[\left(1-\varepsilon_{f, i}^{V}\right) u_{s, i}^{V} N_{i, n}^{\text {share }}\right]}{\sum_{i=1}^{N_{p}}\left[\left(1-\varepsilon_{f, i}^{V}\right) N_{i, n}^{\text {share }}\right]},
$$

7 where $u_{s, i}^{V}$ and $u_{s, n}^{c}$ are the particle velocities in Voronoi cell $i$ and CFD cell $n$, respectively. If $\mathbf{K}_{n}$ represents either the implicit or explicit part of the momentum source term in a CFD cell (Equation 3), and $\mathbf{F}_{i}$ represents the corresponding part of the drag force acting on particle $i$, then the forward mapping of the drag forces from the DEM solver to the CFD solver is

$$
\mathbf{K}_{n}=\sum_{i=1}^{N_{p}}\left(\frac{N_{i, n}^{\text {share }} \mathbf{F}_{i}}{N_{n}^{\text {total }} V_{\text {voro }, i}}\right) .
$$

12 The schematic of the backward mapping (from CFD to DEM) is shown in Fig. 4b. For the field of $\chi_{n}^{c}$,

13 which can be the fluid velocity, pressure gradient force or viscous force, in CFD cell $n$, the mapping scheme adopts a similar logic as the forward step so that

$$
\chi_{i}^{V}=\sum_{n=1}^{N_{f}}\left(\chi_{n}^{C} \frac{N_{i, n}^{\text {share }}}{N_{i}^{\text {total }}}\right) \text {, }
$$
$i$.

Formatted: Font colour: Text 1

Formatted: Font colour: Text 1

Formatted: Font colour: Text 1

Formatted: Font colour: Text 1

Formatted: Font colour: Text 1

Formatted: Font colour: Text 1

Formatted: Font colour: Text 1

Formatted: Font colour: Text 1

Formatted: Font colour: Text 1 
1 To improve the calculation efficiency in both the forward and backward mapping, the field mapping

2 procedure loops over the cells in a linked list (a "neighbour list"), which records the cells in the other

3 grid that have a non-zero $N_{i, n}^{\text {shar }}$ with the target cell (i.e. for each fluid cell there is a neighbour list of

4 Voronoi cells). In this way, less than $1 \%$ of the total number of cells in the other grid are need to be

5 considered for a given target cell.

6

7 In the current version of CPL-Library, only uniform (regular) block meshes are available in the CFD

Formatted: Font colour: Text 1

8 solver (see Fig. 4), and so the field mapping procedures proposed here were implemented and tested for

9 the case of uniform (regular) meshes. However, the generalization of the method proposed here to a

10 non-uniform CFD mesh topology is straight-forward.

11

12 The distance between the neighbouring sampling points should be smaller than the edge length of a

Formatted: Font colour: Text 1

13 hexahedron inscribed in the smallest particle $\left(\theta_{2}<0.577 D_{\min }\right)$, so that each Voronoi cell contains at least

14 one sampling point. The effect of the point cloud density on the field mapping is considered below.

15

16 There are two main advantages to this approach. Firstly, the volume of the Voronoi cell is always larger

17 than the particle, so that all of the CFD and Voronoi cells will have non-zero porosity. Secondly, as the

18 field mapping procedure generates volume-weighted field values using data from several adjacent cells

19 in the other grid, a smooth field can be obtained, which is also a merit of the conventional two-grid

20 methods. 


\section{$1 \quad 3.3$ Simulation data assessment method}

2 To quantitatively assess the porosity field obtained from different coarse-graining methods, three

3 measures are considered. The first is the relative error $(E r)$ of the total particle volume that is mapped

4 onto the CFD grid, $V_{p, \text { total }}^{c}$, to the real solid volume, $V_{p, \text { total, }}$ (i.e. the total volume of particles in the DEM

5 solver),

$$
E r=\frac{V_{p, t o t a l}^{C}-V_{p, t o t a l}}{V_{p, \text { total }}},
$$

8 The second measure is the standard deviation $(S D)$ of the porosity distributions which is defined as

$$
S D=\sqrt{\frac{1}{N_{o c c}-1} \sum_{n=1}^{N_{o c c}}\left(\varepsilon_{f, n}^{C}-\overline{\varepsilon_{f}^{C}}\right)^{2}},
$$

10 where $N_{\text {occ }}$ is the total number of CFD cells that are occupied by the particles. Following [36, 37] the

11 third measure considered is the residual image error $(\gamma)$ which compares the difference between two

12 similar images in terms of pixel intensity level and is given by

13

$$
\gamma=\frac{\left|\hat{\boldsymbol{\varepsilon}}_{f}-\boldsymbol{\varepsilon}_{f}\right|}{\left|\boldsymbol{\varepsilon}_{f}\right|}
$$

14 where $\varepsilon_{f}$ and $\varepsilon_{f}$ are the calculated and the reference image vectors of the porosity, respectively. In images obtained from the calculation.

17

18 The Pearson correlation coefficient $(\rho)$ was introduced to assess the relationship between the individual

19 particle drag forces obtained from different calculation methods. The Pearson correlation coefficient
Formatted: Font colour: Text 1

Formatted: Font colour: Text 1

Formatted: Font colour: Text 1

Formatted: Font colour: Text 1

Formatted: Font colour: Text 1

Formatted: Font colour: Text 1

Formatted: Font colour: Text 1

Formatted: Font colour: Text 1

Formatted: Font colour: Text 1 
1 between two variables $X$ and $Y$ is calculated by [38]

$$
\rho_{X, Y}=\frac{\mathrm{E}[(X-\bar{X})(Y-\bar{Y})]}{S D_{X} S D_{Y}},
$$

4

5 the expectation.

\section{4. Results and discussion}

7 Two typical but contrasting cases were selected to assess the performance of the proposed method. Case

81 is a uniformly packed polydisperse particle assembly with periodic boundaries, this type of assembly

9 can be used to evaluate the fluid-particle interactions in the field of soil mechanics [3, 39]. Case 2 is a

10 spouted fluidised bed, which involves the flow of monodisperse particles with distinctive solid

11 concentration distributions. This type of fluidised bed is frequently seen in the pharmaceutical industry

12 to coat particles $[40]$. The new method proposed here is denoted 2GVM (Two-grid Voronoi Method) and the calculation results are compared to those obtained using PCM and DM [20]

\section{$14 \quad$ 4.1 Validation Case 1: flow through particle assembly}

15 An immersed boundary method (IBM) investigation of fluid-particle interactions in fluid-saturated

16 polydisperse granular materials was carried out by Knight et al $[27]$. One of the cases considered

17 included 497 particles with a linear grading and the particle diameters range from 0.5 to $1.7 \mathrm{~mm}$. To

18 create the samples, particles were randomly placed within cubic periodic boundaries. After that, the

19 sample was subject to increasing isotopic compression up to an effective stress of $100 \mathrm{kPa}$ in the DEM
Formatted: Font colour: Text 1

Formatted: Font colour: Text 1

Formatted: Font colour: Text 1

Formatted: Font colour: Text 1

Formatted: Font colour: Text 1

Formatted: Font colour: Text 1

Formatted: Font colour: Text 1

Formatted: Font colour: Text 1

Formatted: Font colour: Text 1

Formatted: Font colour: Text 1

Formatted: Font colour: Text 1

Formatted: Font colour: Text 1

Formatted: Font colour: Text 1

Formatted: Font colour: Text 1

Formatted: Font colour: Text 1

Formatted: Font colour: Text 1 
1 solver using servo-controlled periodic boundaries [41]. Six snapshots with global porosity values

2 ranging from 0.319 to 0.602 were taken of the system during compression, and the fluid-particle

3 interactions during laminar flow were determined by IBM simulations using the Multiflow code [42,

4 43]. The IBM-generated data is used to validate the proposed 2GVM approach in unresolved CFD-

5 DEM method using the Ergun [7] and Tenneti [9] drag correlations. The Ergun and Tenneti drag

6 expressions were chosen as they previously have been applied to consider this system [44]

\section{〈Table 1 Particle properties and numerical settings in the simulation Case 1>}

Fig. 5 shows the particle assembly along with the CFD grid and Voronoi cells in the coupling. In the

CFD solver, the boundaries in the $y$ and $z$ direction are periodic. In the $x$-direction, the inlet boundary was set to have a fixed fluid velocity and zero pressure gradient, and the outlet was set to a fixed pressure of zero. Periodic boundaries are applied in the DEM solver, and the domain for Voronoi tessellation is restricted to the box that is occupied by the particles. The sample was fixed in space and not moving with the simulation. Table 1 lists the particle properties and numerical settings in the unresolved CFDDEM simulations.

$<$ Fig. 5 Particle assembly in the CFD grid and Voronoi cells $>$

$<$ Fig. 6 Cross-sectional view of the porosity of the particle assembly in the CFD grid $>$

$<$ Fig. 7 Variation of $S D, E r$ and $\gamma$ with $\theta_{2}$.>

$<$ Fig. 8 Histograms of the porosity from different coarse-graining methods. $>$

$<$ Fig. 9 Comparison of the $S D$ of the porosity values and the $E r$ of solid volume for difference CFD grid configurations and coarse-graining methods.>

Fig. 6 shows the porosity distributions on cross-sectional slices in the $x$ - $z$ plane for different CFD grid configurations. The CFD grid configurations considered are $10 \times 10 \times 10,7 \times 7 \times 7$ and $9 \times 1 \times 1$, (number of fluid cells along $x \times$ number of fluid cells along $y \times$ number of fluid cells along $z$ ). These scenarios represent cases where the grid size is slightly smaller than, approximately equal to and larger than the
Formatted: Font colour: Text 1

Formatted: Font colour: Text 1

Formatted: Font colour: Text 1

Formatted: Font colour: Text 1

Formatted: Font colour: Text 1

Formatted: Font colour: Text 1

Formatted: Font colour: Text 1

Formatted: Font colour: Text 1

Formatted: Font colour: Text 1

Formatted: Font colour: Text 1

Formatted: Font colour: Text 1

Formatted: Font colour: Text 1 
1 mean particle size of $1.1 \mathrm{~mm}$. Results from 2GVM are compared with results obtained using PCM and

2 DM. For 2GVM, three different values of $\theta_{2}$ were selected for each grid configuration to investigate the

3 influence of point cloud density.

Formatted: Font colour: Text 1

5 While some conclusions can be drawn from Fig. 6 alone, a comprehensive discussion requires additional

6 analyses. Fig. 7 plots the variation in the $S D, E r$ and $\gamma$ with $\theta_{2}$ for the three CFD grid configurations

7 considered. When $\theta_{2}$ exceeds 3.5 , the SD curves converge to specific values, $E r$ converges to zero, and

$8 \gamma$ converge to a value lower than $3 \%$. These data indicate that, for this system, the porosity fields do not

9 measurably change with any further increase in $\theta_{2}$ for $\theta_{2}>3.5$. All subsequent analysis of this particle

10 assembly is conducted with $\theta_{2}>3.5$.

11

12 Fig. 8 shows the histogram of porosity values obtained from the 2GVM (with $\theta_{2}>3.5$ ), PCM and DM.

13 Consideration of the combined data on Fig. 6 and Fig. 8 shows that the porosity values obtained from

Formatted: Font colour: Text 1

14 the PCM and DM have non-physical porosity values (approximating 0 or 1.0) and large jumps between

Formatted: Font colour: Text 1

15 the neighbouring cells in the $10 \times 10 \times 10$ and $7 \times 7 \times 7$ grid cases. The non-physical features are not

16 observed in the 2 GVM approach and the porosity field becomes smooth, which is consistent with the

17 uniformity of the particle assembly in this case. For the one-dimensional (1D) case $(9 \times 1 \times 1$ grid), each

18 grid cell contains around 42 particles, the effects of coarse-graining approaches on the porosity field are

19 not obvious

$<$ Fig. 10 Local particle porosity against the normalized particle diameter for different coarsegraining methods. The global porosity is indicated by the red line $>$

22 Fig. 9 provides bar charts illustrating the $S D$ of the porosity distributions and the $E r$ of $V_{p, t o t a l}^{C}$ to the real 
1 solid volume. When coarse-graining, the porosity of a CFD cell must be between 0 and 1 . However, in

2 both PCM and DM, the particle volume can exceed the the CFD cell volume if the particle is larger than

3 the CFD cell. In these instances, the excess volume must be discarded to ensure that the porosity lies

4 between 0 and 1, resulting in an error in the total particle volume exchanged with the CFD solver. Fig.

Formatted: Font colour: Text 1

59 shows that $2 \mathrm{GVM}$ gives the lowest $S D$ and $E r$ values amongst the three approaches, and thus the

6 porosity field generated by the $2 \mathrm{GVM}$ is the most uniform and best able to conserve volume. On the

7 other hand, PCM and DM provide results in which volume is conserved only in the $1 \mathrm{D}$ case, in which

8 each CFD grid cell contains more than 40 particles.

$<$ Fig. 11 Individual particle drag forces against diameter at global $\varepsilon_{f}=0.319$ with the drag forces extracted according to PCM, DM and 2 GVM $\left(9 \times 1 \times 1\right.$ CFD grid, $\left.\theta_{2}=3.6\right)$. $>$

$<$ Fig. 12 Individual particle drag forces against those from IBM simulations. The global $\varepsilon_{f}=0.319$.

Note that the data points are transparent - the darker shading indicates overlapping data points.>

$<$ Table 2 Pearson correlation between the individual particle drag forces from the IBM and unresolved CFD-DEM cases. The global $\varepsilon_{f}=0.319$. $>$

Formatted: Font colour: Text 1

Formatted: Font colour: Text 1

Formatted: Font colour: Text 1

Formatted: Font colour: Text 1

In Fig. 10, the local particle porosities calculated from the three coarse-graining methods are plotted

against the normalized diameters for the case where the global porosity is 0.319 . It is clear that as the

particle diameter increases the porosity calculated by the 2GVM decreases significantly, which is to be

expected as (relatively) larger particles are known to experience greater local solid fractions [27]. On

Formatted: Font colour: Text 1

Formatted: Font colour: Text 1

19 the other hand, all the porosities from the PCM and DM are assigned to one of a finite number of fixed

20 levels. This is because each particle is assigned the same porosity value of the specific CFD grid cell in

21 which it is positioned and each cell contains multiple particles.

22

23 Due to the small number of particles in the sample, simulation cases with the PCM and DM could only

24 be reasonably run using the $1 \mathrm{D}$ CFD grid $_{\alpha}(9 \times 1 \times 1)$. For this case, the magnitude of the drag force acting

Formatted: Font colour: Text 1 
1 on each particle normalized by the Stokes force $\left(F_{d}\right)$ is plotted against normalised particle diameter $(D)$

2 in Fig. 11. The local porosity is indicated by the colour of the data points. In Fig. 11, the individual

3 particle drag forces calculated using the PCM and DM approaches are distributed along several straight

4 lines. Each line represents data for particles with the same porosity values (in the same CFD cell). The

5 reason the correlations between $F_{d \Delta}$ and $D_{\Delta}$ are linear can be understood by reference to the analytical

6 forms of the normalised drag forces provided in Knight [44]. At a given porosity, the $F_{d_{4}}$ calculated

7 from the Ergun correlation is linearly proportional to the Reynolds number which in turn is linearly dependant on the particle diameter. The normalised Tenneti drag expression is more complex involving

$9 R e^{0.687}$ and $R e$ terms, and so $F_{d}$ is approximately linearly dependent on the particle diameter when

$100.10<R e<0.33$. In contrast, the drag forces calculated from $2 \mathrm{GVM}$ show a different, nonlinear trend,

11 and are clearly dependent on the particle diameter. The Tenneti correlation provides slightly greater drag

12 forces than those predicted using the Ergun correlation. The individual particle drag forces are plotted

13 against the drag forces obtained from IBM simulations [27] considering the same particle assembly in

14 Fig. 12, the corresponding Pearson correlation values are list in Table 2. The data on Fig. 12 and Table

152 clearly show that the drag force predictions obtained using the $2 \mathrm{GVM}$ more closely follow the trends observed in the IBM data than the predicted values obtained using PCM and DM. Since PCM and DM

21 drag force is estimated by empirical models. Thus, it is not expected to achieve results that exactly agree
Formatted: Font colour: Text 1

Formatted: Font colour: Text 1

Formatted: Font colour: Text 1

Formatted: Font colour: Text 1

Formatted: Font colour: Text 1

Formatted: Font colour: Text 1

Formatted: Font colour: Text 1

Formatted: Font colour: Text 1

Formatted: Font colour: Text 1

Formatted: Font colour: Text 1

Formatted: Font colour: Text 1

Formatted: Font colour: Text 1

Formatted: Font colour: Text 1

Formatted: Font colour: Text 1

Formatted: Font colour: Text 1

Formatted: Font colour: Text 1

Formatted: Font colour: Text 1

Formatted: Font colour: Text 1 
1 with the IBM. However, these data indicate that a significant improvement in the prediction of the drag

2 force on the individual particle can be realised by applying the $2 \mathrm{GVM}$ with a relative computational

3 cost significantly lower than required for resolved CFD-DEM approaches such as the IBM.

$<$ Fig. 13 Local particle drag forces from 2GVM with different mesh densities against the local particle drag forces from IBM simulations. >

$<$ Table 3 Pearson correlation between the individual particle drag force from IBM and unresolved CFD-DEM >

8 Fig. 13 considers the sensitivity of the performance of $2 \mathrm{GVM}$ to packing density and grid configuration,

9 Table 3 lists the corresponding Pearson correlation between the data from 2GVM and IBM. The

10 individual particle drag forces calculated using $2 \mathrm{GVM}$ are plotted against the local particle drag forces

Formatted: Font colour: Text 1

11 from IBM simulations for 4 porosity values ranging from 0.319 to 0.602 . For each porosity considered,

12 data obtained with grid densities of $4 \times 4 \times 4,7 \times 7 \times 7$ and $10 \times 10 \times 10$ are compared. In all cases the Ergun

13 drag correlation was applied. Relatively low Pearson correlation values can be seen in the cases with

14 low global porosities, which indicates the prediction is less accurate in the case of densely packed

Formatted: Font colour: Text 1

15 particle assemblies. This can most likely be attributed to the fact that the overall porosity of densely

16 packed case is slightly out of the recommended scope of the Ergun $\left(0.320 \leq \varepsilon_{f} \leq 0.470\right)$ and Tenneti

$17\left(0.350 \leq \varepsilon_{f} \leq 0.90\right)$ expressions which have an empirical basis $[44]$. For cases with the same global porosity,

18 the distribution of the drag force from different CFD grid densities are in close agreement, thus data

Formatted: Font colour: Text 1

Formatted: Font colour: Text 1

19 from CFD-DEM simulations using the 2GVM are relatively independent of the CFD grid density.

$<$ Fig. 14 Variation in volume averaged drag force with global porosity. $>$

21 The overall drag force applied to the assembly was divided by the total volume of the assembly to obtain

22 the volume-averaged drag force which is shown in Fig. 14. The results calculated from different

23 approaches are in a close agreement. Combing Fig. 11, Fig. 12 and Fig. 14, it can be concluded that the 
$12 \mathrm{GVM}$ provides excellent prediction in the individual particle drag force and the overall drag force of

2 the particle assembly. The PCM and DM provide a good prediction of the overall drag force of the

3 particle assembly even though the data presented above showed they are unable to predict individual

4 particle drag forces correctly. Therefore, $2 \mathrm{GVM}$ is a better choice in the case of polydisperse systems,

5 to ensure that relative particle motion is accurately captured.

\section{$6 \quad 4.2$ Validation Case 2: Spouted fluidised bed}

7 To evaluate the ability of $2 \mathrm{GVM}$ to predict the dynamic mechanisms associated with fluidisation

8 processes, the second validation case considered published experimental data for a pseudo-2D spouted

9 fluidised bed documented in [20]. Fig. 15 shows the dimensions of the targeted spouted fluidised bed,

10 which has two inlets and one outlet. The inner inlet and outer inlet introduce the upward fluidisation air

11 for spout fluidisation and background fluidisation, respectively. CFD grids with two densities, i.e., a

12 coarse and a fine grid, were used. Each CFD cell contains around 2 and 25 particles for the fine and

13 coarse grid cases, respectively. $2.5 \mathrm{~mm}$ diameter glass beads were used in these experiments. The particle

14 properties $[20]$ and numerical settings for the simulations are listed in Table 4. In these simulations, the drag force model proposed in Gidaspow [8] is employed, which applies the Wen and Yu [32] and Ergun

$16[7]$ drag correlations for the dilute $\left(\varepsilon_{f}>0.8\right)$ and dense $\left(\varepsilon_{f}<0.8\right)$ regions, respectively. The Ergun and

17 Wen and Yu models are used as they have been widely used to simulate the fluidization process $[45]$.

<Table 4 Particle properties and numerical settings in the simulation Case 2>

19 In contrast to validation Case 1, there are regions of very low packing density in this system and so a

20 bounding cuboid was applied to each particle and the sides of the Voronoi cells were not allowed to

Formatted: Font colour: Text 1

Formatted: Font colour: Text 1

Formatted: Font colour: Text 1

Formatted: Font colour: Text 1

Formatted: Font colour: Text 1

Formatted: Font colour: Text 1

Formatted: Font colour: Text 1

Formatted: Font colour: Text 1

Formatted: Font colour: Text 1

Formatted: Font colour: Text 1

Formatted: Font colour: Text 1

Formatted: Font colour: Text 1

Formatted: Font colour: Text 1 
1 extend beyond the cuboid boundaries, as illustrated for a representative particle distribution in Fig. 16a.

3 The effectiveness of different coarse-graining approaches in capturing the spatial distribution of porosity

4 is shown in Fig. 16b and c for the fine and coarse grid configurations. 2GVM produces a substantially

5 more smoother porosity field, which is most evident when considering the fine grid in Fig. 16b.

6 Quantitative assessment of the differences between 2GVM, DM and PCM are presented in Fig. 17 and

7 Fig. 18. Fig. 17 shows the variation in SD and $\gamma$ for increasing $\theta_{1}$, while Fig. 18 shows the variation in

$8 S D, E r$ and $\gamma$ with increasing $\theta_{2}$. This analysis indicates that the spatial distribution of porosity is

Formatted: Font colour: Text 1

9 insensitive to changes for $\theta_{1}>3.0$ and $\theta_{2}>3.0$, which is in alignment with the observations for validation

10 Case 1. By comparing Fig. 7a with Fig. 18a, it is clear that the two figures indicate different initial trends

Formatted: Font colour: Text 1

11 in the $S D$ variation with $\theta_{2}$. These differences arise because when $\theta_{2}$ is small $\left(\theta_{2} \precsim 1.5\right)$, the point cloud

12 density is low, therefore not all of the Voronoi cells may be detected by the point cloud. The resulting

13 porosity field does not then correctly represent the true solid distribution, and the $E r$ in the total particle

14 volume is high (see Fig.18b). In these scenarios, the $S D$ of the porosity field can be either higher (Fig.

$157 \mathrm{a}$ ) or lower (Fig. 18a) than the final values $\left(\theta_{2}>3.0\right)$, resulting in different trends in the $S D$ curves.

16 These data confirm that the method is not effective if a $\theta_{2}$ value $<1.5$ is selected.

17

18 In order to compare the porosity fields calculated from 2GVM, PCM and DM, Fig. 19 shows the

19 histogram of the porosity from these methods, while the $S D$ and $E r$ of the porosity values are shown in

20 Fig. 20. Only the CFD cells occupied by particles were included in the statistics. From Fig. 19, the

21 distributions of the porosity predicted from PCM and DM are not continuous for the fine CFD grid case, 
1 as indicated by the relatively large jumps that exist between cells. In addition, particle volume is not

2 conserved when PCM is used with a fine grid (see Fig. 20). The nonuniformity and jumps in the porosity

3 field from PCM and DM can be confirmed by the visual inspection of Fig. 16. A coarse CFD grid

4 produces more similar results between the various methods. As the CFD grid size is increased, the three

5 methods provide SD values for the porosity field that are very close to each other and the total solid

6 volume is now effectively conserved in all cases $(E r \approx 0)$. Owing to the low cell-to-particle size ratio in

7 the fine grid case, the accuracy of porosity field predicted by PCM and DM is low. By a similar logic,

8 the differences in the porosity distributions estimated from the three coarse-graining methods are not

9 expected to be pronounced for the coarse grid case. The above trends were also observed in validation

10 Case 1.

11 Fig. 16d shows the porosity differences, which are obtained by subtracting the porosity values in each

Formatted: Font colour: Text 1

12 CFD cell obtained using the PCM and DM from the 2GVM values, for the fine and coarse CFD grid

13 cases. From Fig. 16d, the porosity differences in the fine CFD grid case are evident in the dilute particle

14 region between the solid and fluid phase. For the coarse CFD grid case, the magnitude of the image

15 difference is obviously lower and is uniformly distributed in the region that occupied by the particles.

16 As mentioned above, PCM and DM have limitations when calculating porosity for the fine CFD grid

17 but are suitable for the coarse CFD grid cases, 2GVM is suitable for both fine and coarse CFD grid

18 cases. Accordingly, Fig. 16d indicates that 2GVM mainly improves the porosity field in the dilute flow

19 regions of fine CFD grid case.

${ }_{4}<$ Fig. 15 The dimensions and grid of the spouted fluidised bed $>$

Formatted: Font colour: Text 1 
$<$ Fig. 17 Variation of SD and $\gamma$ with $\theta_{1}\left(\theta_{2}=3.0\right)$ for the coarse CFD grid $>$

$<$ Fig. 18 Variation of $S D, E r$ and $\gamma$ with $\theta_{2}\left(\theta_{1}=3.0\right)>$

$<$ Fig. 19 Histogram distributions of the porosity values in CFD grid from different coarse-graining methods.>

$<$ Fig. 20 Comparison of the $S D$ of the porosity distributions from different coarse-graining methods $>$ $<$ Fig. 21 Comparison of the particle flux profiles from the experiment and simulations $>$

7 Fig. 21 compares the particle flux profiles obtained from the experimental image analysis of the CCD

camera and the CFD-DEM simulations. The particle flux is the averaged value over 5 seconds of

9 physical time in the simulations. A good agreement is observed for all of the simulation cases. In

10 validation Case 1 , we were able to demonstrate that $2 \mathrm{GVM}$ both provides a smooth porosity field as

11 well as a correct estimation of the individual particle drag forces at the particle-scale. For this validation

12 case, only the particle flow information at the macro-level (such as the particle flux) are extracted from

13 experimental measurements and available for the comparison with numerical simulation. The advantage

14 of $2 \mathrm{GVM}$ is not obvious in such conditions as the conventional PCM and DM are able to give a reliable

15 prediction of the macro-level flow dynamics. The objective of the current study was to validate /

16 benchmark the methods using data from a well-constrained problem for which reliable experimental

17 data are available. Future studies will consider application of the 2GVM model to polydisperse systems

18 involving fluidised beds.

\section{5. Conclusions}

Formatted: Font colour: Text 1

20 This study introduced a new coarse-grained approach for CFD-DEM simulations (2GVM). The new

21 approach used a radical Voronoi tessellation so that the fluid-particle interaction force can be calculated

22 using local porosity data. A point cloud is used to map field variables between the CFD and DEM solvers.

23 Two different, but typical validation cases were used to evaluate the performance of the proposed 
1 method.

2 The following main conclusions are drawn based on the two verification studies considered:

3 1. The new coarse-graining method (2GVM) conserves data, is grid-independent and can generate smooth porosity field in the CFD side without using any smoothing or thresholding method.

52 2. $2 \mathrm{GVM}$ provides a more reasonable estimation of the individual particle porosity for polydisperse systems, and results in an accurate prediction on the individual particle drag when compared with data obtained using a fully resolved IBM simulations.

8 3. The approach taken to modify the Voronoi tessellation with an outer bounding cuboid can be easily applied to the applications of the fluidised bed, where the solid concentration is non-uniformly distributed.

11 This new coarse-graining approach is particularly relevant to cases where the variation in the fluid-

12 particle interaction force with particle size is important, for example in the simulation of internal instability in the embankment soils or any application where particle segregation occurs.

\section{Acknowledgements}

15 This work was financially supported by EPSRC grant EP/P010393/1. Key development work by Dr.

16 Edward Smith was funded by the embedded CSE programme of the ARCHER UK National

17 Supercomputing Service (http://www.archer.ac.uk).

\section{References}


1 Apparatus, Advances in Chemical Engineering, Academic Press2015, pp. 83-135.

2 [2] T. Han, A. Levy, H. Kalman, DEM simulation for attrition of salt during dilute-phase pneumatic

3 conveying, Powder Technology 129 (2003) 92-100.

4 [3] Z. Hu, Y. Zhang, Z. Yang, Suffusion-induced deformation and microstructural change of granular soils: a 5 coupled CFD-DEM study, Acta Geotechnica 14 (2019) 795-814.

6 [4] Z.Y. Zhou, S.B. Kuang, K.W. Chu, A.B. Yu, Discrete particle simulation of particle-fluid flow: model

7 formulations and their applicability, Journal of Fluid Mechanics 661 (2010) 482-510.

8 [5] C.S. Peskin, The immersed boundary method, Acta numerica 11 (2002) 479-517.

9 [6] T.B. Anderson, R. Jackson, Fluid Mechanical Description of Fluidized Beds. Equations of Motion,

10 Industrial \& Engineering Chemistry Fundamentals 6 (1967) 527-539.

11 [7] S. Ergun, Fluid Flow Through Packed Columns, Fluid Flow Through Packed Columns (1952) 89-94.

12 [8] D. Gidaspow, Multiphase flow and fluidization: continuum and kinetic theory descriptions, Academic 13 press1994.

14 [9] S. Tenneti, R. Garg, S. Subramaniam, Drag law for monodisperse gas-solid systems using particle15 resolved direct numerical simulation of flow past fixed assemblies of spheres, International Journal of $16 \quad$ Multiphase Flow 37 (2011) 1072-1092.

17 [10] Y. Tang, E.A.J.F. Peters, J.A.M. Kuipers, S.H.L. Kriebitzsch, M.A. van der Hoef, A new drag correlation

18 from fully resolved simulations of flow past monodisperse static arrays of spheres, AIChE Journal 61 (2015)

$19 \quad 688-698$.

20 [11] C. Kloss, C. Goniva, A. Hager, S. Amberger, S. Pirker, Models, algorithms and validation for opensource 21 DEM and CFD-DEM, Progress in Computational Fluid Dynamics, an International Journal 12 (2012) 140- 
$1 \quad 152$

2 [12] R. Sun, H. Xiao, Diffusion-based coarse graining in hybrid continuum-discrete solvers: Theoretical

3 formulation and a priori tests, International Journal of Multiphase Flow 77 (2015) 142-157.

4 [13] D.A. Clarke, A.J. Sederman, L.F. Gladden, D.J. Holland, Investigation of Void Fraction Schemes for

5 Use with CFD-DEM Simulations of Fluidized Beds, Industrial \& Engineering Chemistry Research 57 (2018)

$6 \quad 3002-3013$.

7 [14] Y. Tsuji, T. Kawaguchi, T. Tanaka, Discrete particle simulation of two-dimensional fluidized bed, Powder $8 \quad$ Technology 77 (1993) 79-87.

9 [15] Z. Peng, E. Doroodchi, C. Luo, B. Moghtaderi, Influence of void fraction calculation on fidelity of CFD-

10 DEM simulation of gas-solid bubbling fluidized beds, AIChE Journal 60 (2014) 2000-2018.

11 [16] C.L. Wu, J.M. Zhan, Y.S. Li, K.S. Lam, A.S. Berrouk, Accurate void fraction calculation for three-

12 dimensional discrete particle model on unstructured mesh, Chemical Engineering Science 64 (2009) 1260-

$13 \quad 1266$

14 [17] Z. Peng, B. Moghtaderi, E. Doroodchi, A modified direct method for void fraction calculation in CFD-

15 DEM simulations, Advanced Powder Technology 27 (2016) 19-32.

16 [18] T. Tsuji, K. Higashida, Y. Okuyama, T. Tanaka, Fictitious particle method: A numerical model for flows

17 including dense solids with large size difference, AIChE Journal 60 (2014) 1606-1620.

18 [19] L. Jing, C.Y. Kwok, Y.F. Leung, Y.D. Sobral, Extended CFD-DEM for free-surface flow with multi-size

19 granules, International Journal for Numerical and Analytical Methods in Geomechanics 40 (2016) 62-79.

20 [20] J.M. Link, L.A. Cuypers, N.G. Deen, J.A.M. Kuipers, Flow regimes in a spout-fluid bed: A combined

21 experimental and simulation study, Chemical Engineering Science 60 (2005) 3425-3442. 
1 [21] B.J. Glasser, I. Goldhirsch, Scale dependence, correlations, and fluctuations of stresses in rapid granular

2 flows, Physics of Fluids 13 (2001) 407-420.

3 [22] S. Deb, D.K. Tafti, A novel two-grid formulation for fluid-particle systems using the discrete element

4 method, Powder Technology 246 (2013) 601-616.

5 [23] J. Su, Z. Gu, C. Chen, X.Y. Xu, A two-layer mesh method for discrete element simulation of gas-particle

6 systems with arbitrarily polyhedral mesh, International Journal for Numerical Methods in Engineering 103

$7 \quad(2015) 759-780$.

8 [24] B.J. Gellatly, J.L. Finney, Characterisation of models of multicomponent amorphous metals: The radical

9 alternative to the Voronoi polyhedron, Journal of Non-Crystalline Solids 50 (1982) 313-329.

10 [25] C.H. Rycroft, VORO++: A three-dimensional Voronoi cell library in C++, Chaos: An Interdisciplinary

11 Journal of Nonlinear Science 19 (2009) 041111.

12 [26] G. Frenning, Efficient Voronoi volume estimation for DEM simulations of granular materials under 13 confined conditions, MethodsX 2 (2015) 79-90.

14 [27] C. Knight, C. O'Sullivan, B. van Wachem, D. Dini, Computing drag and interactions between fluid and

15 polydisperse particles in saturated granular materials, Computers and Geotechnics 117 (2020) 103210.

16 [28] R. Sun, H. Xiao, SediFoam: A general-purpose, open-source CFD-DEM solver for particle-laden flow 17 with emphasis on sediment transport, Computers \& Geosciences 89 (2016) 207-219.

18 [29] C. Goniva, C. Kloss, A. Hager, S. Pirker, An open source CFD-DEM perspective, Proceedings of

19 OpenFOAM Workshop, Göteborg, 2010, pp. 22-24.

20 [30] C. Kloss, C. Goniva, G. Aichinger, S. Pirker, Comprehensive DEM-DPM-CFD simulations-model 21 synthesis, experimental validation and scalability, Proceedings of the seventh international conference on 
1 CFD in the minerals and process industries, CSIRO, Melbourne, Australia, 2009, pp. 9-11.

2 [31] E.R. Smith, D.J. Trevelyan, E. Ramos-Fernandez, A. Sufian, C. O’Sullivan, D. Dini, CPL library - A

3 minimal framework for coupled particle and continuum simulation, Computer Physics Communications

4 (2019) 107068.

5 [32] C.Y. Wen, Y.H. Yu, A generalized method for predicting the minimum fluidization velocity, AIChE

6 Journal 12 (1966) 610-612.

7 [33] R.I. Issa, Solution of the implicitly discretised fluid flow equations by operator-splitting, Journal of

8 Computational Physics 62 (1986) 40-65.

9 [34] P.A. Cundall, Strack, A Discrete numerical model for granular assemblies, International Journal of Rock

10 Mechanics and Mining Sciences \& Geomechanics Abstracts 29 (1979) 47-65.

11 [35] H.Q. Che, D. Liu, W.B. Tian, S. Gao, J.T. Sun, L.J. Xu, CFD-DEM study of gas-solid flow regimes in a

12 Wurster type fluidized bed with experimental validation by electrical capacitance tomography, Chemical

13 Engineering Journal 389 (2020) 124280.

14 [36] C.G. Xie, S.M. Huang, C.P. Lenn, A.L. Stott, M.S. Beck, Experimental evaluation of capacitance

15 tomographic flow imaging systems using physical models, IEE Proceedings - Circuits, Devices and Systems

$16141(1994) 357-368$.

17 [37] W. Yang, L. Peng, Image reconstruction algorithms for electrical capacitance tomography, Measurement

18 Science and Technology 14 (2002) R1.

19 [38] C. Zaiontz, Real Statistics Using Excel: Correlation, Basic Concepts, Real Statistics 2020,

20 http://www.real-statistics.com/correlation/basic-concepts-correlation/, 2020.

21 [39] X. Huang, Exploring Critical-state Behaviour Using DEM, Imperial College London London, 2014. 
1 [40] L.A.P. de Freitas, Pharmaceutical applications of spouted beds: A review on solid dosage forms,

$2 \quad$ Particuology 42 (2019) 126-136.

3 [41] C. Thornton, Numerical simulations of deviatoric shear deformation of granular media, Géotechnique $450(2000) 43-53$.

5 [42] F. Denner, B.G.M. van Wachem, Fully-Coupled Balanced-Force VOF Framework for Arbitrary Meshes

6 with Least-Squares Curvature Evaluation from Volume Fractions, Numerical Heat Transfer, Part B:

$7 \quad$ Fundamentals 65 (2014) 218-255

8 [43] M.H. Abdol Azis, F. Evrard, B. van Wachem, An immersed boundary method for incompressible flows

$9 \quad$ in complex domains, Journal of Computational Physics 378 (2019) 770-795.

10 [44] C. Knight, Fluid flow and drag in polydisperse granular materials subject to laminar seepage flow,

11 Imperial College London, 2018.

12 [45] H.P. Zhu, Z.Y. Zhou, R.Y. Yang, A.B. Yu, Discrete particle simulation of particulate systems: Theoretical

13 developments, Chemical Engineering Science 62 (2007) 3378-3396.

14

Formatted: Font colour: Text 1 
2 Figure list:

3 Fig. 1 Schematic of the conventional and radical Voronoi tessellation

4 Fig. 2 CFD-DEM coupling in the CPL-Library

5 Fig. 3 Schematic of the Voronoi tessellation of the particle system (the Voronoi cells are built in 3D space)

6 Fig. 4 Schematic of the field mapping method using point cloud

$7 \quad$ Fig. 5 Particle assembly in the CFD grid and Voronoi cells

8 Fig. 6 Cross-sectional view of the porosity of the particle assembly in the CFD grid.

$9 \quad$ Fig. 7 Variation of $S D, E r$ and $\gamma$ with $\theta_{2}$.

10 Fig. 8 Histograms of the porosity from different coarse-graining methods.

11 Fig. 9 Comparison of the $S D$ of the porosity values and the $E r$ of solid volume for difference CFD grid

12 configurations and coarse-graining methods.

13 Fig. 10 Local particle porosity against the normalized particle diameter for different coarse-graining

14 methods. The global porosity is indicated by the red line

15 Fig. 11 Individual particle drag forces against diameter at global $\varepsilon_{f}=0.319$ with the drag forces extracted

16 according to PCM, DM and $2 \mathrm{GVM}\left(9 \times 1 \times 1\right.$ CFD grid, $\left.\theta_{2}=3.6\right)$.

Formatted: Font colour: Text 1

17 Fig. 12 Individual particle drag forces against those from IBM simulations. The global $\varepsilon_{f}=0.319$. Note that

18 the data points are transparent - the darker shading indicates overlapping data points.

19 Fig. 13 Local particle drag forces from 2GVM with different mesh densities against the local particle drag

20 forces from IBM simulations.

21 Fig. 14 Variation in volume averaged drag force with global porosity.

22 Fig. 15 The dimensions and grid of the spouted fluidised bed

23 Fig. 16 Plots of the same solids distribution with different coarse-graining methods.

24 Fig. 17 Variation of SD and $\gamma$ with $\theta_{1}\left(\theta_{2}=3.0\right)$ for the coarse CFD grid

25 Fig. 18 Variation of SD, Er and $\gamma$ with $\theta_{2}\left(\theta_{1}=3.0\right)$

26 Fig. 19 Histogram distributions of the porosity values in CFD grid from different coarse-graining methods.

27 Fig. 20 Comparison of the $S D$ of the porosity distributions from different coarse-graining methods

28 Fig. 21 Comparison of the particle flux profiles from the experiment and simulations 


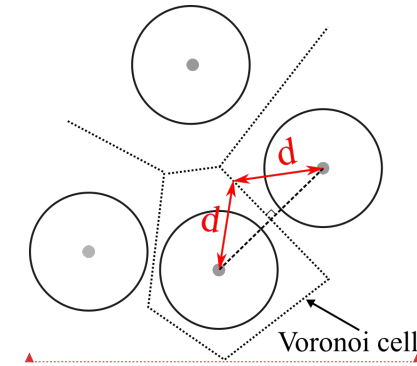

(a) Conventional Voronoi tessellation

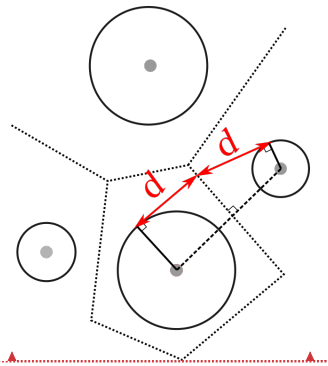

(b) Radical Voronoi tessellation

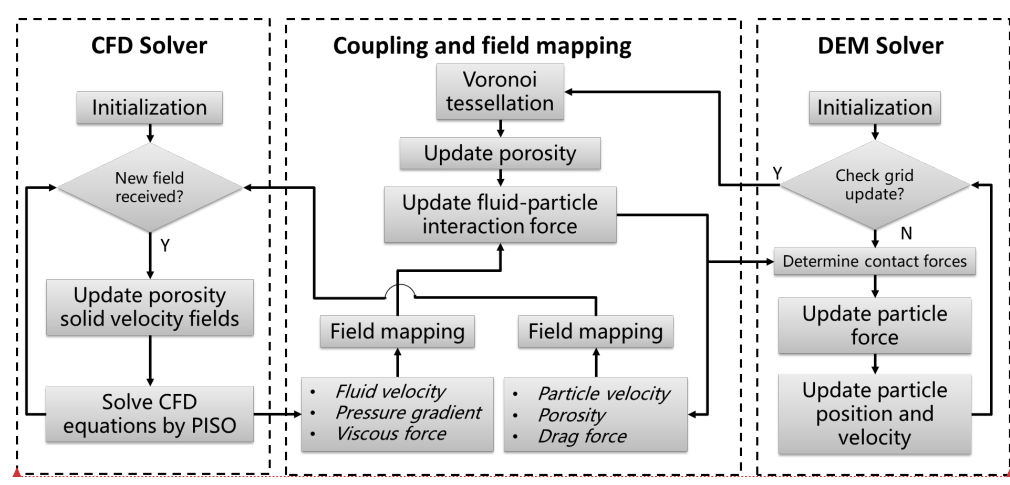

Fig. 2 CFD-DEM coupling in the CPL-Library

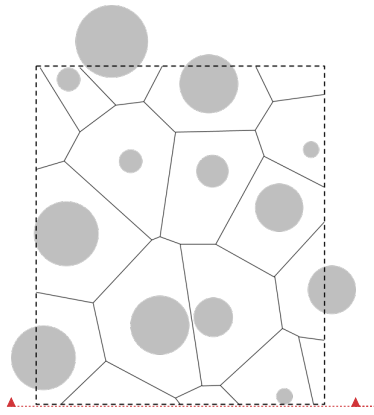

(a) Uniform particle packing

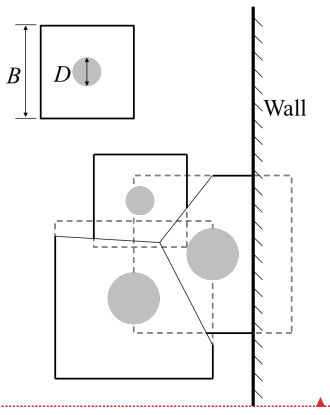

(b) Dilute particle flow region

Fig. 3 Schematic of the Voronoi tessellation of the particle system (the Voronoi cells are built in 3D space)

Formatted: Font colour: Text 1

Formatted: Font colour: Text 1

Formatted: Font colour: Text 1

Formatted: Font colour: Text 1

Formatted: Font colour: Text 1

Formatted: Font colour: Text 1

Formatted: Font colour: Text 1

Formatted: Font colour: Text 1

Formatted: Font colour: Text 1

Formatted: Font colour: Text 1

Formatted: Font colour: Text 1

Formatted: Font colour: Text 1

Formatted: Font colour: Text 1 


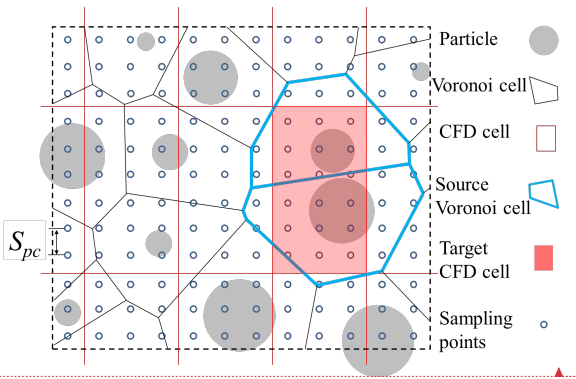

(a) Field mapping from Voronoi cells to a CFD cell

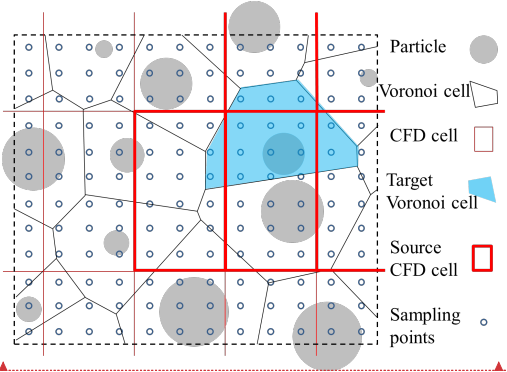

(b) Field mapping from CFD cells to a Voronoi

cell

Fig. 4 Schematic of the field mapping method using point cloud

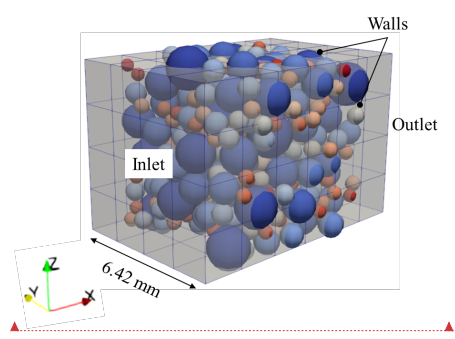

(a) Particle assembly in the CFD grid

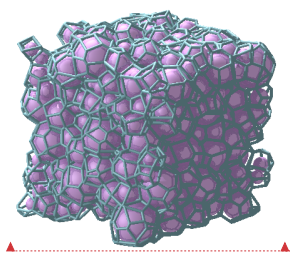

(b) Voronoi tessellation of the particle assembly
Formatted: Font colour: Text 1

Formatted: Font colour: Text 1

Formatted: Font colour: Text 1

Formatted: Font colour: Text 1

Formatted: Font colour: Text 1

Formatted: Font colour: Text 1

Formatted: Font colour: Text 1

Formatted: Font colour: Text 1

Formatted: Font colour: Text 1

Formatted: Font colour: Text 1

Formatted: Font colour: Text 1

Formatted: Font colour: Text 1

Formatted: Font colour: Text 1

Formatted: Font colour: Text 1 


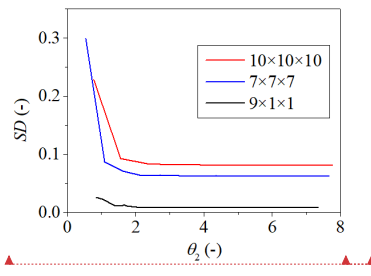

(a) Standard deviation of the porosity field

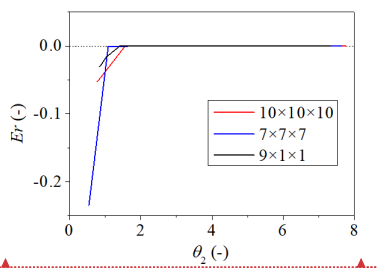

(b) Relative error in overall solid volume

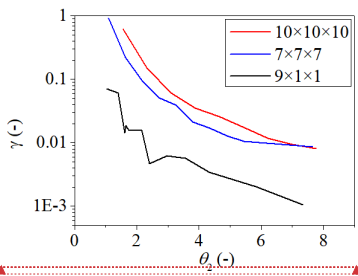

(c) Residual image error of the cross-sectional porosity

Fig. 7 Variation of $S D, E r$ and $\gamma$ with $\theta_{2}$.

Formatted: Font colour: Text 1

Formatted: Font colour: Text 1

Formatted: Font colour: Text 1

Formatted: Font colour: Text 1

Formatted: Font colour: Text 1

Formatted: Font colour: Text 1

Formatted: Font colour: Text 1

Formatted: Font colour: Text 1

$10 \times 10 \times 10$ grid:

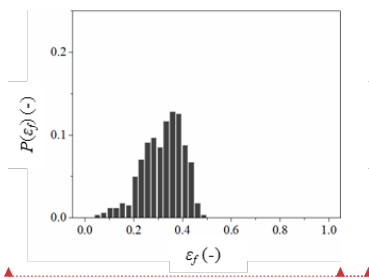

$2 \mathrm{GVM}\left(\theta_{2}=3.9\right)$

$7 \times 7 \times 7$ grid:

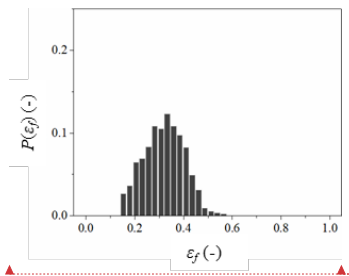

$2 \mathrm{GVM}\left(\theta_{2}=3.8\right)$

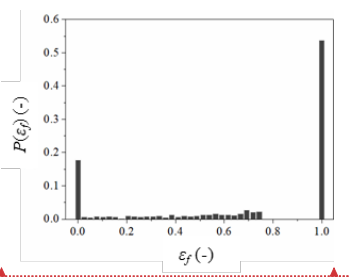

$\varepsilon_{f}(-)$

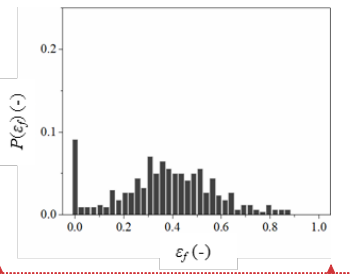

DM

PCM

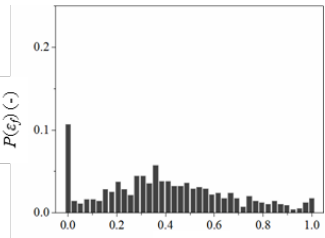

Formatted: Font colour: Text 1

Formatted: Font colour: Text 1

Formatted: Font colour: Text 1

Formatted: Font colour: Text 1

Formatted: Font colour: Text 1

Formatted: Font colour: Text 1

Formatted: Font colour: Text 1

Formatted: Font colour: Text 1

Formatted: Font colour: Text 1

Formatted: Font colour: Text 1

Formatted: Font colour: Text 1

Formatted: Font colour: Text 1

Formatted: Font colour: Text 1

Formatted: Font colour: Text 1

Formatted: Font colour: Text 1
Formatted: Font colour: Text 1
DM

PCM

methods. 
Fig. 9 Comparison of the $S D$ of the porosity values and the $E r$ of solid volume for difference CFD

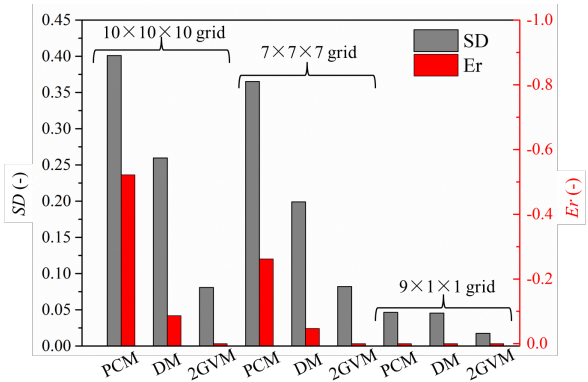

Formatted: Font colour: Text 1

Formatted: Font colour: Text 1

grid configurations and coarse-graining methods.

7

8
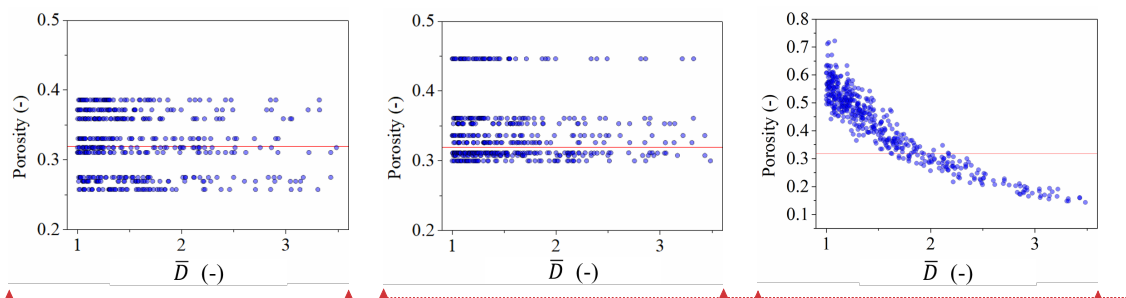

Formatted: Font colour: Text 1

Formatted: Font colour: Text 1

Formatted: Font colour: Text 1

Formatted: Font colour: Text 1

Formatted: Font colour: Text 1

Formatted: Font colour: Text 1

(a) $\mathrm{PCM}$

(b) DM

(c) $2 \mathrm{GVM}$

Formatted: Font colour: Text 1

Fig. 10 Local particle porosity against the normalized particle diameter for different coarse-graining methods. The global porosity is indicated by the red line

Formatted: Font colour: Text 1

11

12

13 

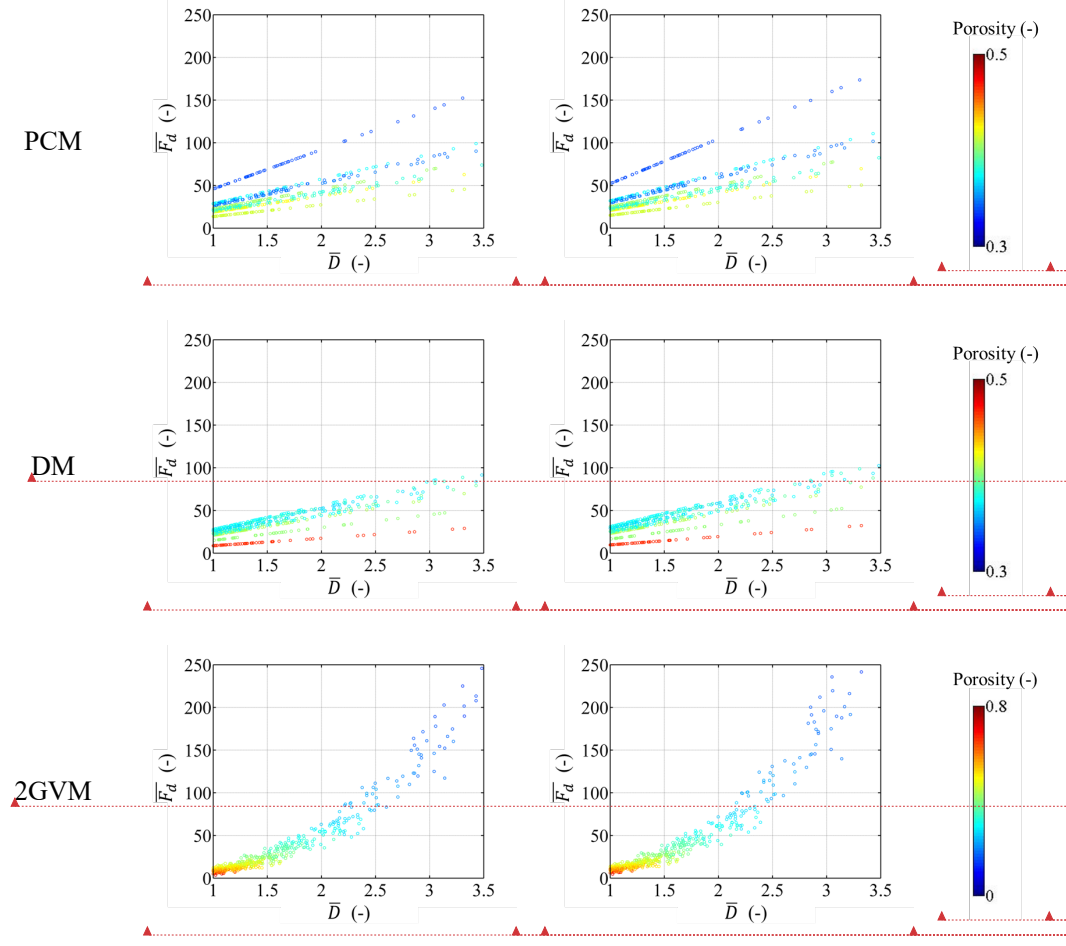

Ergun
Formatted: Font colour: Text 1

Formatted: Font colour: Text 1

Formatted: Font colour: Text 1

Formatted: Font colour: Text 1

Formatted: Font colour: Text 1

Formatted: Font colour: Text 1

Formatted: Font colour: Text 1

Formatted: Font colour: Text 1

Formatted: Font colour: Text 1

Formatted: Font colour: Text 1

Formatted: Font colour: Text 1

Formatted: Font colour: Text 1

Formatted: Font colour: Text 1

Formatted: Font colour: Text 1

Formatted: Font colour: Text 1

Formatted: Font colour: Text 1

Formatted: Font colour: Text 1

Formatted: Font colour: Text 1

Formatted: Font colour: Text 1

Formatted: Font colour: Text 1

Formatted: Font colour: Text 1

Formatted: Font colour: Text 1 

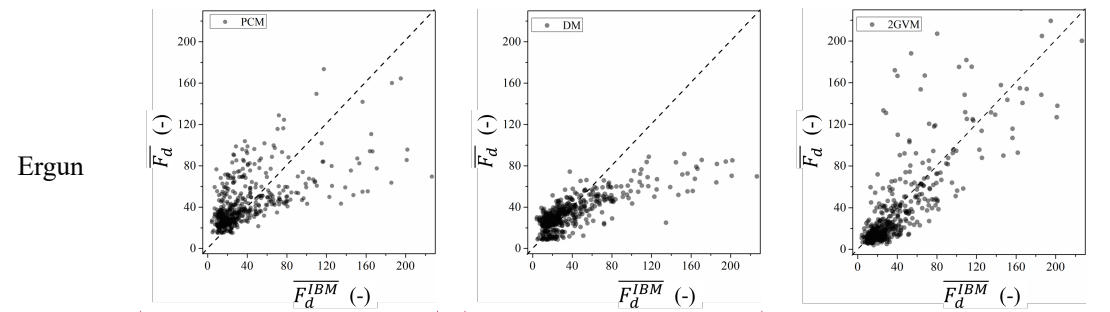

\begin{tabular}{l} 
Formatted: Font colour: Text 1 \\
Formatted: Font colour: Text 1 \\
Formatted: Font colour: Text 1 \\
Formatted: Font colour: Text 1 \\
Formatted: Font colour: Text 1 \\
\hline
\end{tabular}
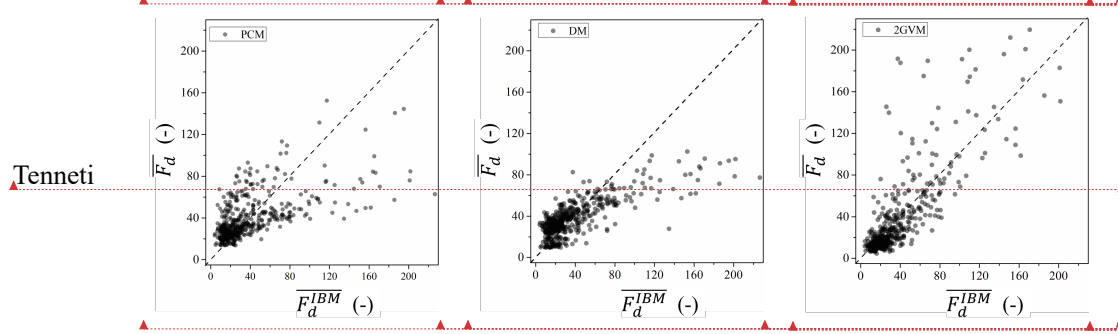

Formatted: Font colour: Text 1

Formatted: Font colour: Text 1

Formatted: Font colour: Text 1

Formatted: Font colour: Text 1

Formatted: Font colour: Text 1

Formatted: Font colour: Text 1

Formatted: Font colour: Text 1

PCM

DM

2GVM

Formatted: Font colour: Text 1

Fig. 12 Individual particle drag forces against those from IBM simulations. The global $\varepsilon_{f}=0.319$. Note that the data points are transparent - the darker shading indicates overlapping data points. 
1
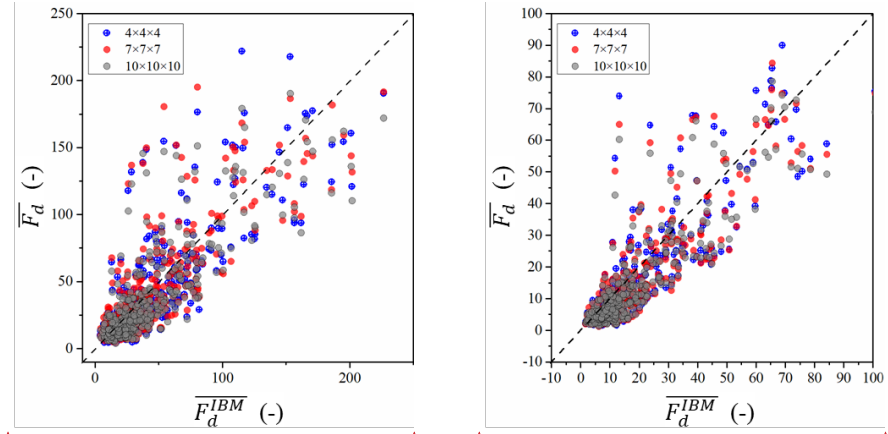

Formatted: Font colour: Text 1

Formatted: Font colour: Text 1

Formatted: Font colour: Text 1

Formatted: Font colour: Text 1

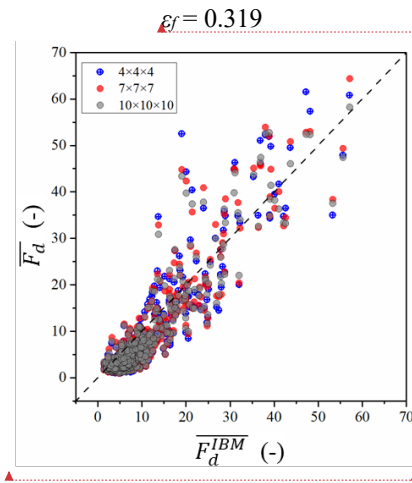

$\varepsilon_{f}=0.478$

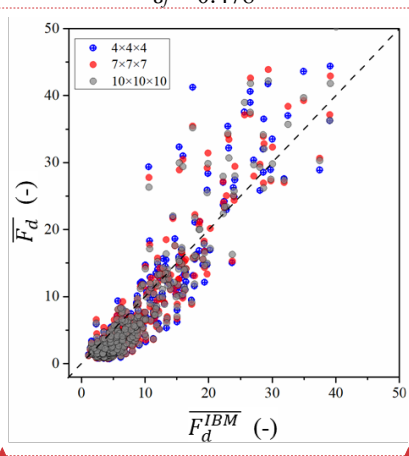

Formatted: Font colour: Text 1

Formatted: Font colour: Text 1

Formatted: Font colour: Text 1

Formatted: Font colour: Text 1

Formatted: Font colour: Text 1

$$
\varepsilon_{f}=0.551
$$

$\varepsilon_{f}=0.602$

Formatted: Font colour: Text 1

Fig. 13 Local particle drag forces from 2GVM with different mesh densities against the local particle drag forces from IBM simulations.
Formatted: Font colour: Text 1 


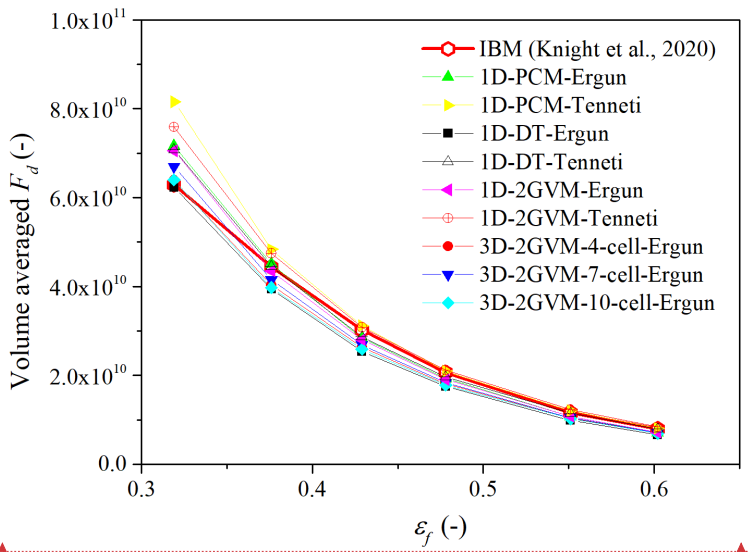

Formatted: Font colour: Text 1

Formatted: Font colour: Text 1

Fig. 14 Variation in volume averaged drag force with global porosity

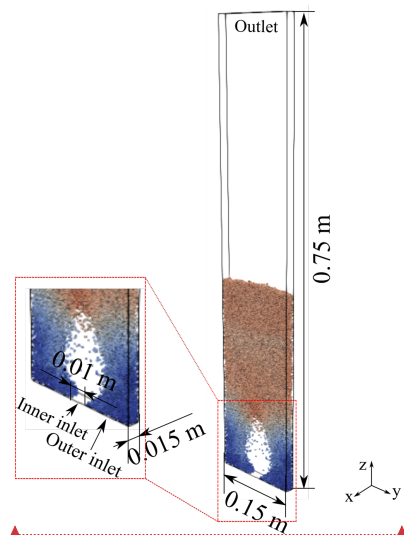

Formatted: Font colour: Text 1

Formatted: Font colour: Text 1

Fig. 15 The dimensions and grid of the spouted fluidised bed
5 


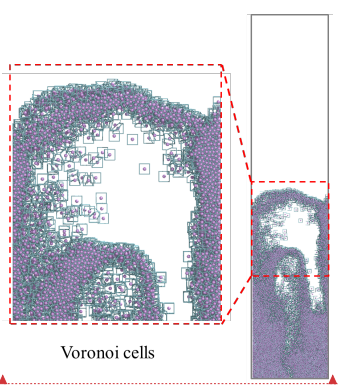

(a) The real particle distribution $\left(\theta_{1}=3.0\right)$

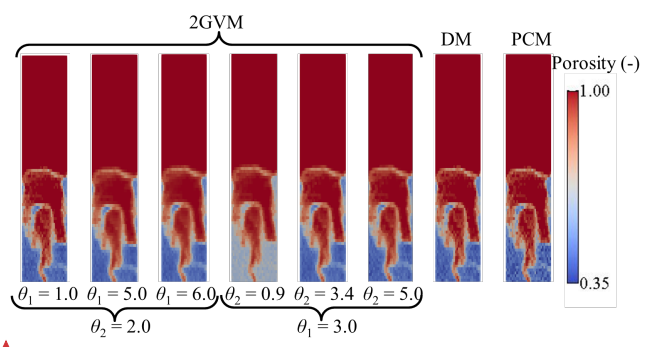

(c) Porosity distributions for coarse CFD gird

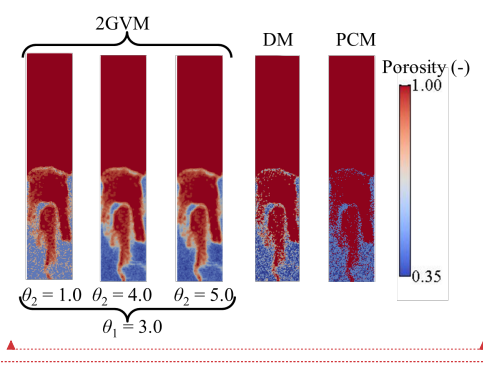

(b) Porosity distributions for fine CFD gird

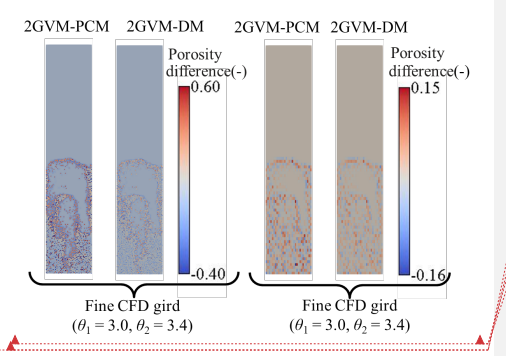

(d) Difference between porosity values calculated using $2 \mathrm{GVM}$ and those obtained using PCM \& DM

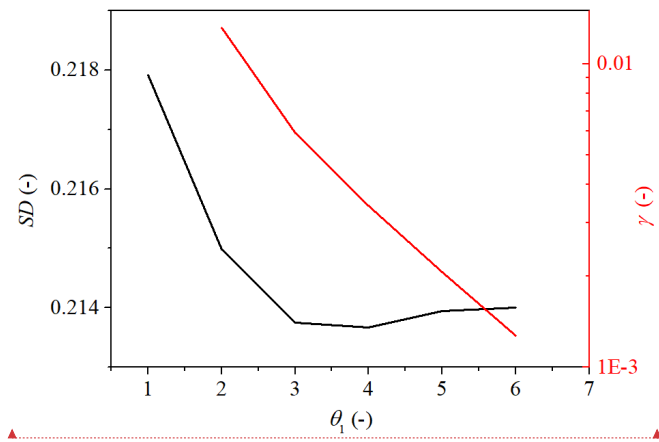

Formatted: Font colour: Text 1

Formatted: Font colour: Text 1

Fig. 17 Variation of SD and $\gamma$ with $\theta_{1}\left(\theta_{2}=3.0\right)$ for the coarse CFD grid 
1

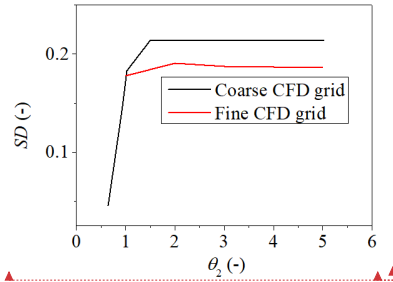

(a) Standard deviation

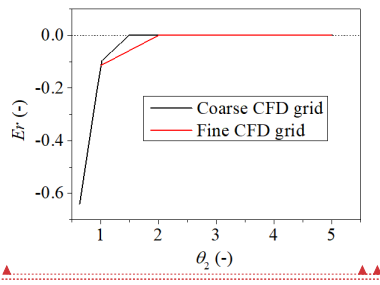

(b) Relative error

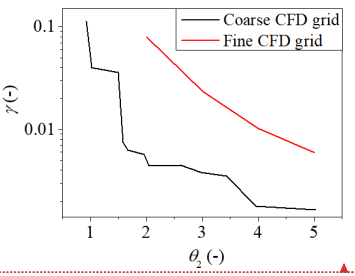

(c) Residual image error

Formatted: Font colour: Text 1

Formatted: Font colour: Text 1

Formatted: Font colour: Text 1

Formatted: Font colour: Text 1

Formatted: Font colour: Text 1

Formatted: Font colour: Text 1

Formatted: Font colour: Text 1

Formatted: Font colour: Text 1

Fine CFD grid:

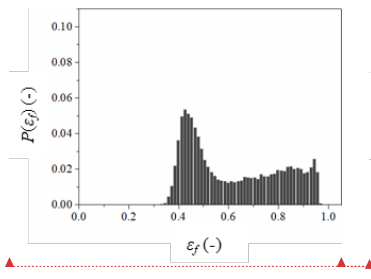

$2 \mathrm{GVM}\left(\theta_{1}=3.0, \theta_{2}=3.0\right)$

Coarse CFD grid:
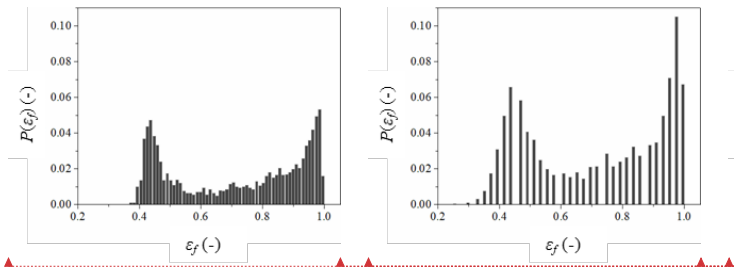

$2 \operatorname{GVM}\left(\theta_{1}=3.0, \theta_{2}=2.0\right)$

Fig. 19 Histogram distributions of the porosity values in CFD grid from different coarse-graining methods.

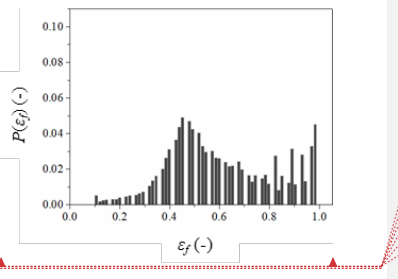

DM

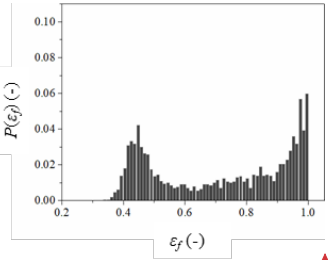

DM
Formatted: Font colour: Text 1

Formatted: Font colour: Text 1

Formatted: Font colour: Text 1

Formatted: Font colour: Text 1

Formatted: Font colour: Text 1

Formatted: Font colour: Text 1

Formatted: Font colour: Text 1

Formatted: Font colour: Text 1

Formatted: Font colour: Text 1

Formatted: Font colour: Text 1

Formatted: Font colour: Text 1

Formatted: Font colour: Text 1

Formatted: Font colour: Text 1

Formatted: Font colour: Text 1

Formatted: Font colour: Text 1

Formatted: Font colour: Text 1 
1

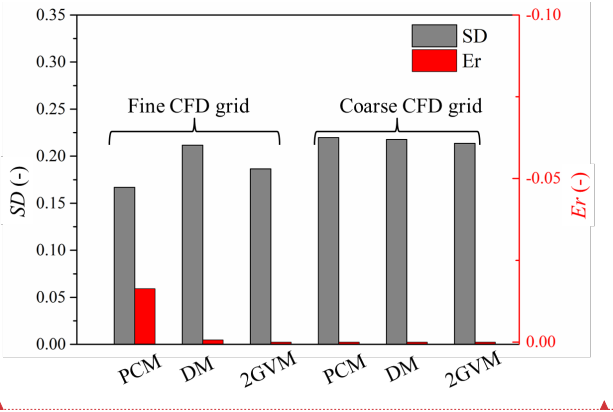

Fig. 20 Comparison of the $S D$ of the porosity distributions from different coarse-graining methods

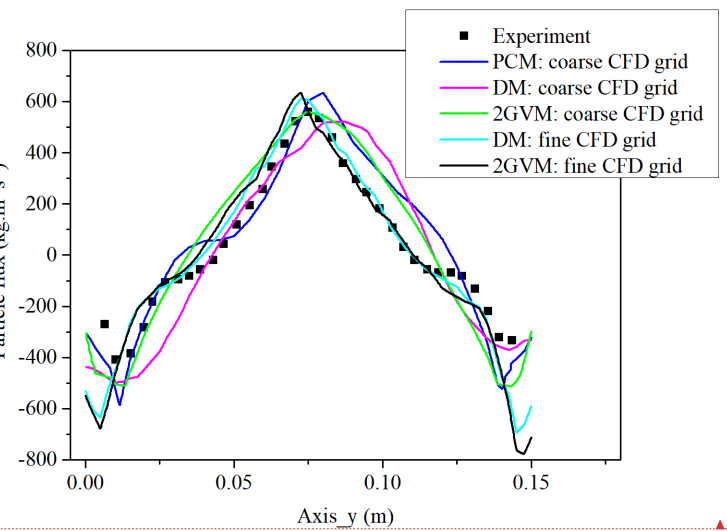

Fig. 21 Comparison of the particle flux profiles from the experiment and simulations
Formatted: Font colour: Text 1

Formatted: Font colour: Text 1

Formatted: Font colour: Text 1

Formatted: Font colour: Text 1 
2 Table list:

3 Table 1 Particle properties and numerical settings in the simulation Case 1

4 Table 2 Pearson correlation between the individual particle drag forces from the IBM and unresolved CFD-

$5 \quad$ DEM cases. The global $\varepsilon_{f}=0.319$.

6 Table 3 Pearson correlation between the individual particle drag force from IBM and unresolved CFD-

7 DEM

$8 \quad$ Table 4 Particle properties and numerical settings in the simulation Case 2

Formatted: Font colour: Text 1

Table 1 Particle properties and numerical settings in the simulation Case 1

\begin{tabular}{lll} 
Property & Value & Unit \\
\hline Number of particles $\left(N_{p}\right)$ & 497 & - \\
Density $\left(\rho_{p}\right)$ & 2470 & $\mathrm{~kg} / \mathrm{m}^{3}$ \\
Diameter $(D)$ & $0.5 \sim 1.7$ & $\mathrm{~mm}$ \\
Coefficient of restitution $(e)$ & 0.95 & - \\
Coulomb friction coefficient $(\mu)$ & 0.3 & - \\
Inlet velocity $(U)$ & $2 \times 10^{-4}$ & $\mathrm{~m} / \mathrm{s}$ \\
Time step of CFD solver $\left(\Delta t_{1}\right)$ & $5 \times 10^{-6}$ & $\mathrm{~s}$ \\
Time step of DEM solver $\left(\Delta t_{2}\right)$ & $5 \times 10^{-8}$ & $\mathrm{~s}$ \\
Coupling interval $(M)$ & & \\
Point cloud density $\left(\theta_{2}\right)$ & $100 \Delta t_{1}$ & $\mathrm{~s}$
\end{tabular}

Formatted: Font colour: Text 1

Formatted: Font colour: Text 1

Formatted: Font colour: Text 1

Formatted: Font colour: Text 1

Formatted: Font colour: Text 1

Formatted: Font colour: Text 1

Formatted: Font colour: Text 1

Formatted: Font colour: Text 1

Formatted: Font colour: Text 1

Formatted: Font colour: Text 1

Formatted: Font colour: Text 1

Formatted: Font colour: Text 1 
4 Table 2 Pearson correlation between the individual particle drag forces from the IBM and unresolved 5 CFD-DEM cases. The global $\varepsilon_{f}=0.319$.

\begin{tabular}{lccc}
\hline Drag correlation & PCM & DM & 2GVM \\
\hline Ergun & 0.61 & 0.78 & 0.82 \\
Tenneti & 0.62 & 0.78 & 0.81 \\
\hline
\end{tabular}

7

9 Table 3 Pearson correlation between the individual particle drag force from IBM and unresolved

10 CFD-DEM

\begin{tabular}{ccccc}
\hline Grid density & $\varepsilon_{f}=0.319$ & $\varepsilon_{f}=0.478$ & $\varepsilon_{f}=0.551$ & $\varepsilon_{f}=0.602$ \\
\hline $4 \times 4 \times 4$ & 0.83 & 0.86 & 0.91 & 0.92 \\
$7 \times 7 \times 7$ & 0.83 & 0.87 & 0.92 & 0.93 \\
$10 \times 10 \times 10$ & 0.84 & 0.88 & 0.92 & 0.93 \\
\hline
\end{tabular}

11

12

13

14

15 
Table 4 Particle properties and numerical settings in the simulation Case 2

\begin{tabular}{|c|c|c|}
\hline Property & Value & Unit \\
\hline Particle number $\left(N_{p}\right)$ & 24500 & - \\
\hline Density $\left(\rho_{p}\right)$ & 2505 & $\mathrm{~kg} / \mathrm{m}^{3}$ \\
\hline Diameter $(D)$ & 2.5 & $\mathrm{~mm}$ \\
\hline coefficient of normal restitution $(e)$ & 0.97 & - \\
\hline Coulomb friction coefficient $(\mu)$ & 0.1 & - \\
\hline Coarse CFD grid & $2 \times 30 \times 80$ & - \\
\hline Fine CFD grid & $6 \times 60 \times 300$ & - \\
\hline Inner inlet velocity $\left(U_{\mathrm{sp}}\right)$ & 30.0 & $\mathrm{~m} / \mathrm{s}$ \\
\hline Outer inlet velocity $\left(U_{\mathrm{bg}}\right)$ & 1.5 & $\mathrm{~m} / \mathrm{s}$ \\
\hline Cuboid-to-particle size ratio $\left(\theta_{1}\right)$ & $1 \sim 6$ & - \\
\hline Point cloud density $\left(\theta_{2}\right)$ & $1 \sim 5$ & - \\
\hline Time step of CFD solver $\left(\Delta t_{1}\right)$ & $5 \times 10^{-5}$ & $\mathrm{~s}$ \\
\hline Time step of DEM solver $\left(\Delta t_{2}\right)$ & $5 \times 10^{-7}$ & $\mathrm{~s}$ \\
\hline Coupling interval $(M)$ & $100 \Delta t_{1}$ & $\mathrm{~s}$ \\
\hline
\end{tabular}

Formatted: Font colour: Text 1

Formatted: Font colour: Text 1

Formatted: Font colour: Text 1

Formatted: Font colour: Text 1

Formatted: Font colour: Text 1

Formatted: Font colour: Text 1

Formatted: Font colour: Text 1

Formatted: Font colour: Text 1

Formatted: Font colour: Text 1

Formatted: Font colour: Text 1

Formatted: Font colour: Text 1

Formatted: Font colour: Text 1

Formatted: Font colour: Text 1 